\title{
Microstructure and microtexture evolution of shear bands in Al-Cu single crystal during asymmetric rolling
}

\author{
Xiaobin Guo a , Yunlai Deng ${ }^{\text {a,b,*, Yong Zhang }}{ }^{\text {a,b }}$, Jin Zhang ${ }^{\text {b,c }}$, Xinming Zhang ${ }^{\text {a,b }}$ \\ ${ }^{\text {a }}$ School of Materials Science and Engineering, Central South University, Changsha, China \\ ${ }^{\mathrm{b}}$ State Key Laboratory of High Performance and Complex Manufacturing, Central South University, \\ Changsha, China \\ ${ }^{\mathrm{c}}$ Light Alloy Research Institute, Central South University, Changsha, China \\ *Corresponding author. Tel.: +86 731 88876913. E-mail address: luckdeng@csu.edu.cn.
}

Abstract: Asymmetric rolling process (ASR) introduces extra shear deformation compared with conventional symmetric rolling (SR), and induces shear bands in microstructures. ASR technique has been applied to manufacture thick aluminum plate with improved mechanical properties due to the fine grains or even ultrafine-grains obtained in shear bands. Single crystal specimens of Al-Cu alloys were prepared in this work to study the microstructure and texture evolution in asymmetric rolled sheet. Microstructures of deformation bands were observed and the microtexture was analyzed through electron backscatter diffraction (EBSD). Compared with the SR process, ASR induced more homogeneous deformation and shear bands were observed in deformation area. Microstructure of shear bands depends on the initial orientations of single crystals. After single crystal with $(3,-1,9)$ plane orientation is processed in ASR, shear bands consist of S-type grains were formed, which have uniform size and almost the same twisting degree. But for single crystal with $(-3,0,7)$ plane orientation, shear bands have various twisting degrees and grains distributed inhomogeneously. Texture of the shear bands in ASR processed specimens always rotated towards Copper component for single crystals with different orientations.

Keywords: Asymmetric rolling; Single crystal; EBSD; Shear bands; Texture

\section{Introduction}

Asymmetric rolling process (ASR), which was applied to obtain fine grains or even 
ultrafine-grained materials, can feasibly be adapted in industry scale [1-2]. As previous investigations reported [3-5], asymmetric rolling is a process in which the upper and lower rolls with different peripheral velocities or feeding the sheet into the rotating rolls with a specified angle. A rolling technique combined the rolls with different peripheral velocities and horizontally-displaced rolls to get the specified feeding angle has been presented as snake rolling [6]. Compared with conventional symmetric rolling (SR) process, ASR introduces severe plastic deformation and extra shear bands appear in microstructures. The snake rolling technique has been applied to manufacture thick aluminum plate with improved mechanical properties due to the extra shear deformation introduced compared with conventional symmetric process [7-8].

Textures formed by ASR process are different from those formed by SR process [9-10]. In metals with face-centered cubic (f.c.c) crystal structure, such as aluminum, for SR process, the brass-type component, $\{110\}<112>$ increases with rolling reduction, but copper-type, $\{112\}<111>$ and S-type, $\{123\}<634>$ reach the maximum at reduction greater than $80 \%$ [11]. The development of texture component is equal to the $\beta$ fiber change in the Euler space, which runs from brass through $S$ to copper [11]. However, the additional shear deformation in ASR process produces a strong $\{111\} \|$ ND fiber texture [9]. While aluminum alloys with $\{111\}$ fiber texture component have lower planar anisotropy [12], Al sheets manufactured by ASR can present high formability [7].

Single crystals have been used in analyzing texture because the deformation texture developed by rolling depends on the initial crystal orientation [13-16]. As the single crystallographic orientation of single crystal, the slight difference of microstructure and texture components is more easily to observe, when single crystals were used in studying the various rolling process. Kikuchi et al. [14] studied the rotation of rolling textures in copper single crystals, and they found that $\{112\}<111>,\{110\}<112>$, $\{110\}<001>$ and $\{123\}<412>$ orientations are maintained after heavy deformation. However, crystal rotation occurs easily in the case of single crystals with the initial 
$\{123\}<412>$ and $\{001\}<100>$ orientations. All the above texture results were based on texture measurements without reference to microstructure observations or on qualitative estimates of the lattice rotations leading to the textures. Rolling textures change of specimens is easy to test and analyze quantitatively combined with the microstructure observation by the electron backscatter diffraction (EBSD) method. In this study, single crystal specimens of Al-Cu alloys were processed by ASR and SR. Microstructures of deformation bands were observed and the microtexture evolution was analyzed through EBSD.

\section{Materials and Methods}

The raw material of the single crystal specimens used in this study was $\mathrm{Al}-1.45 \mathrm{Cu}$ (wt. \%) alloy. Single crystal specimens with grain up to $10 \mathrm{~mm}$ diameter were grown by pulling bars cut from the alloy plate to $0.5 \sim 1.0 \%$ strain, followed by annealing in a forced-air furnace at $525^{\circ} \mathrm{C}$ for 24 hours, then repeating the cycle $8 \sim 10$ times. Single crystals 1\#, 2\# and 3\# were cut from the macro-grains bars showed in Fig. 1. The three dimensions of all three rectangular specimens are the same with $15 \mathrm{~mm} \times 10 \mathrm{~mm}$ $\times 10 \mathrm{~mm}$. The orientations of three single crystals were determined by EBSD in the FEI Helios NanoLab ${ }^{\text {TM }}$ 600i DualBeam Scanning Electron Microscopy. Crystallographic orientations of raw single crystals $1 \#$ and 2\# are the same with $(3,-1$, 9) $[-8,3,3]$, while $3 \#$ has a different orientation $(-3,0,7)[7,-5,3]$. Single-pass cold-rolling experiments were carried out on a special rolling system, which is showed as schematic in Fig. 1. The rolling mill has a roll diameter of $100 \mathrm{~mm}$ for both rolls, at room temperature and without lubricants applied, and the rotational speed of the lower roll was fixed at $15 \mathrm{rpm}$. The speed ratio $\left(\omega_{1} / \omega_{2}\right)$ of two rolls could be adjusted through change the rotational speed of upper roll, while an offset distance $(\Delta \mathrm{S})$ also could be set. For ASR process in this study, the rotational ratio between the upper and lower rolls $\left(\omega_{1} / \omega_{2}\right)$ is 1.3 and the offset distance $(\Delta S)$ is $4 \mathrm{~mm}$. For comparison, conventional SR process (i.e. $\omega_{1} / \omega_{2}=1$ and $\Delta S=0$ ) experiments is conducted. Single crystals 2\# and 3\# were under ASR process, while 1\# was SR. All three single crystals were cold-rolled by $50 \%$ reduction. Texture and microstructure of 
rolled sheets were measured by EBSD, and all the microstructures were recorded on the normal plane (ND) of the rolled sheet. Prior to EBSD, the normal rolling surface (RD-TD) of the samples were mechanically ground and polished to 3000 grit using $\mathrm{SiC}$ paper, then electro-polishing in a mixture of $10 \%$ perchloric acid and $90 \%$ ethanol at $22 \mathrm{~V}$ for $10 \mathrm{~s}$ at room temperature. The raw data were analyzed using the TSL OIM Analysis 6.5 soft ware [17]. Microtextures were determined according to only the originally indexed points from the raw EBSD detected data. Grain size of specimens were determined by the statistic average diameter in TSL OIM Analysis 6.5 soft ware.
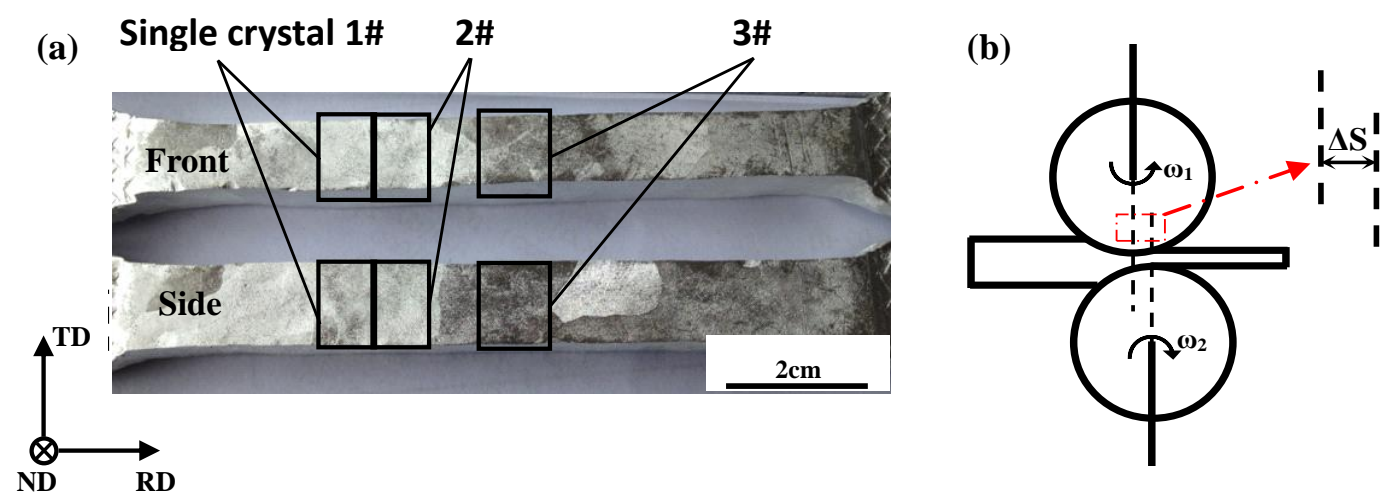

Fig. 1. (a) Large grains bar with single crystal specimens 1\#, 2\# and 3\#; (b) Schematic illustration of the rolling system designed for ASR process.

\section{Results}

Crystallographic orientations of raw single crystals $1 \#$ and $2 \#$ are the same, while 3\# has a different orientation. Fig. 2a, b and c show representative EBSD micrographs (inverse pole figure maps) of the raw single crystals $1 \#$, 2\# and 3\#, respectively. Microstructures of single crystal specimens after ASR (Fig. 3b and 3c) were much different from specimens processed in SR process (Fig. 3a). It is obvious that deformation in ASR is more homogeneous compared with the SR process, of which inhomogeneous deformation leads to the stress concentration area that couldn't be detected in EBSD. IPF maps of the rectangular areas in Fig. 3 show that shear bands appeared after ASR, while no grain rotations appeared in deformation area after SR 
process. The grain boundaries in Fig. 3 were defined as low angle grains with $2^{\circ}-5^{\circ}$, sub-grains with $5^{\circ}-15^{\circ}$, and high angle grains with $15^{\circ}-180^{\circ}$, showed in blue, red and yellow colors respectively.

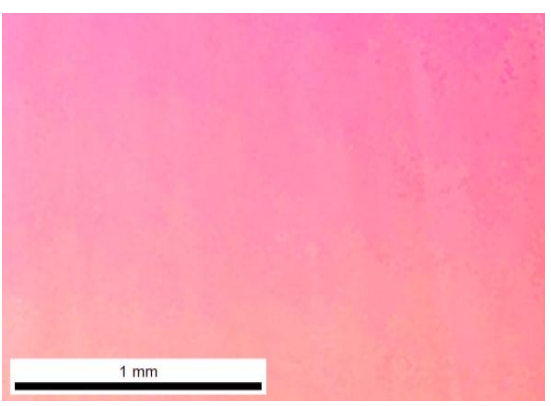

(a)

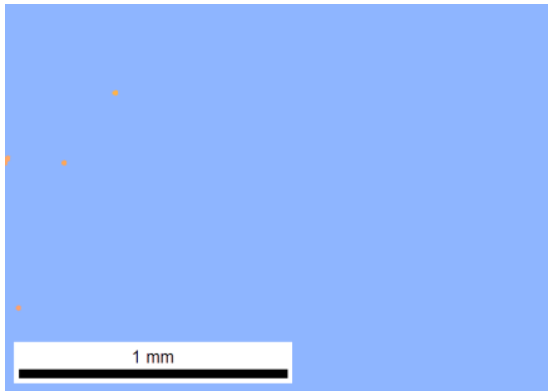

(c)

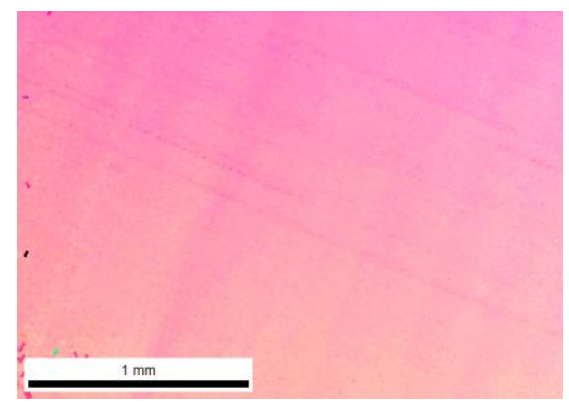

(b)

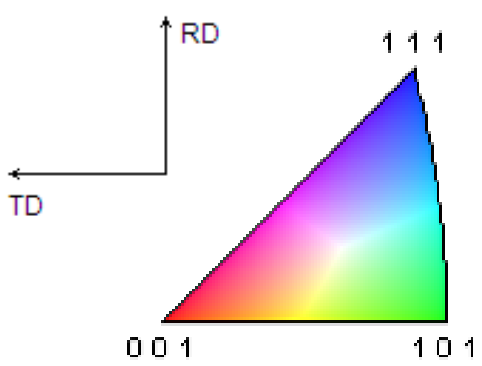

(d)

Fig. 2. The orientations of the $\mathrm{Al}-2 \mathrm{Cu}$ alloys single crystal specimens were determined by EBSD, (a) of $1 \#$ specimen and (b) of $2 \#$ specimen with (hkl) [uvw] orientation of $(3,-1,9)[-8,3,3]$; (c) of $3 \#$ specimen with (hkl) [uvw] orientation of $(-3,0,7)$ [7, -5, 3]; (d) Color coded map type of the [001] IPF in aluminum.

Microstructures of shear bands in Fig. $3 \mathrm{~b}$ and $\mathrm{c}$ are not quite the same, which indicated that structure of deformation area depends on the crystallographic orientation of raw material. After 2\# single crystal is processed in ASR, shear bands consist of S-type grains were formed, which have uniform size and almost the same twisting degree, as shown in Fig. 3b. While in 3\# specimen, shear bands have various twisting degrees and grains distributed inhomogeneously after ASR.

Grain sizes of three specimens after being rolled were measured, the statistical results is shown in Fig. 4. As the initial materials are single crystals, what we concern is the grain size of deformation area. In Fig. 4, the peak value of horizontal ordinate represents the grain size in deformation bands. Grain sizes of $2 \#$ specimen with $(3,-1$, 9) plane orientation in raw material is $5.8 \mu \mathrm{m}$, which is the smallest in three rolled 
specimens. When the plane orientation of raw material changed to $(-3,0,7)$, namely 3\# specimen, the grain size after ASR is $14.1 \mu \mathrm{m}$, which is even larger than SR processed 1\# specimen with $8.1 \mu \mathrm{m}$ of grain size in deformation area.
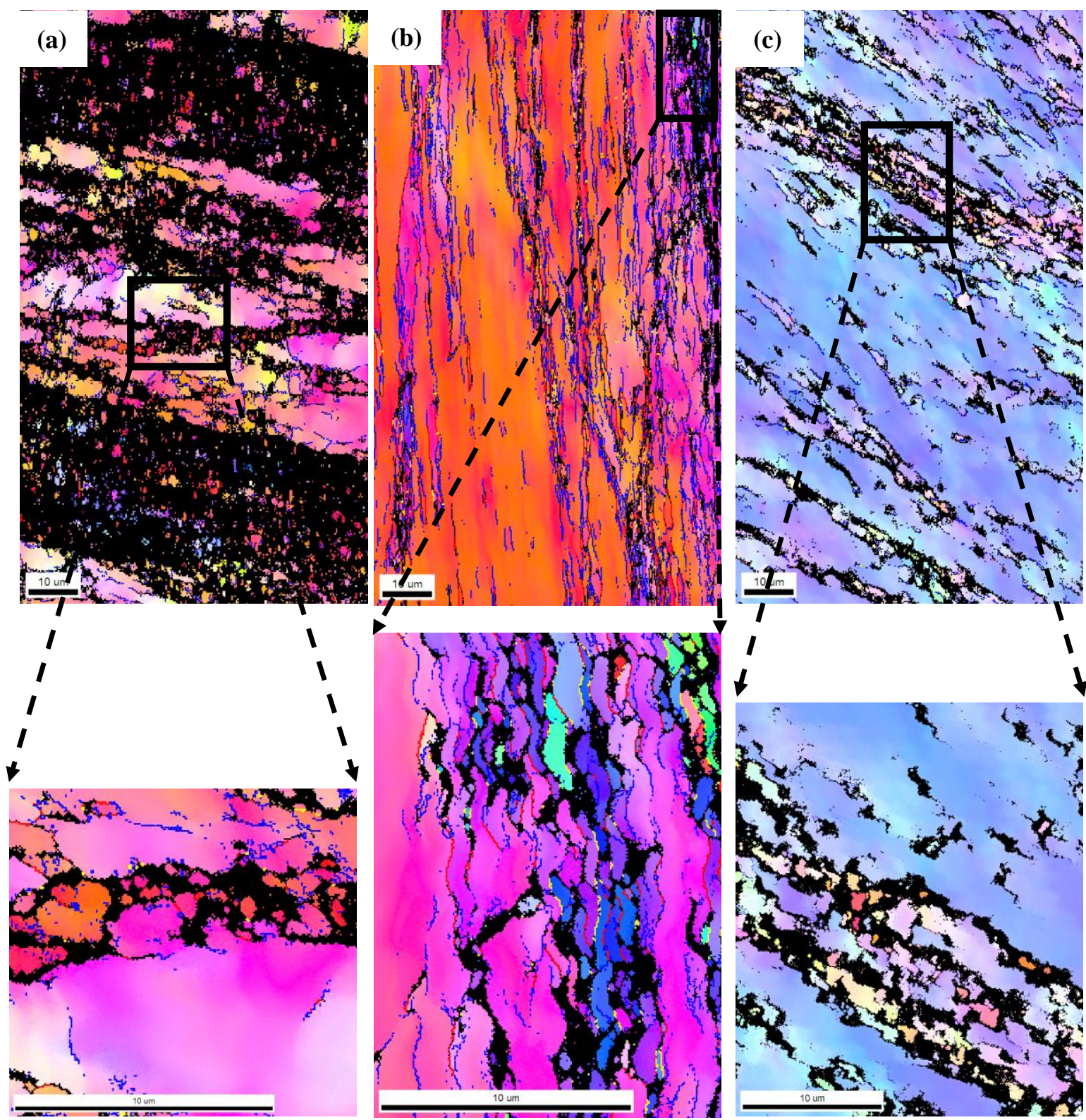

Fig. 3. ND-IPF maps for the three $\mathrm{Al}-2 \mathrm{Cu}$ alloys single crystal specimens processed in different rolling conditions: (a) 1\# specimen processed in SR; (b) 2\# specimen processed in ASR; (c) 3\# specimen processed in ASR. Rectangular areas were shown more clearly under the IPF maps. The grain boundaries were defined as low angle grains with $2^{\circ}-5^{\circ}$, sub-grains with $5^{\circ}-15^{\circ}$, and high angle grains with $15^{\circ}-180^{\circ}$, showed in blue, red and yellow colors respectively. 


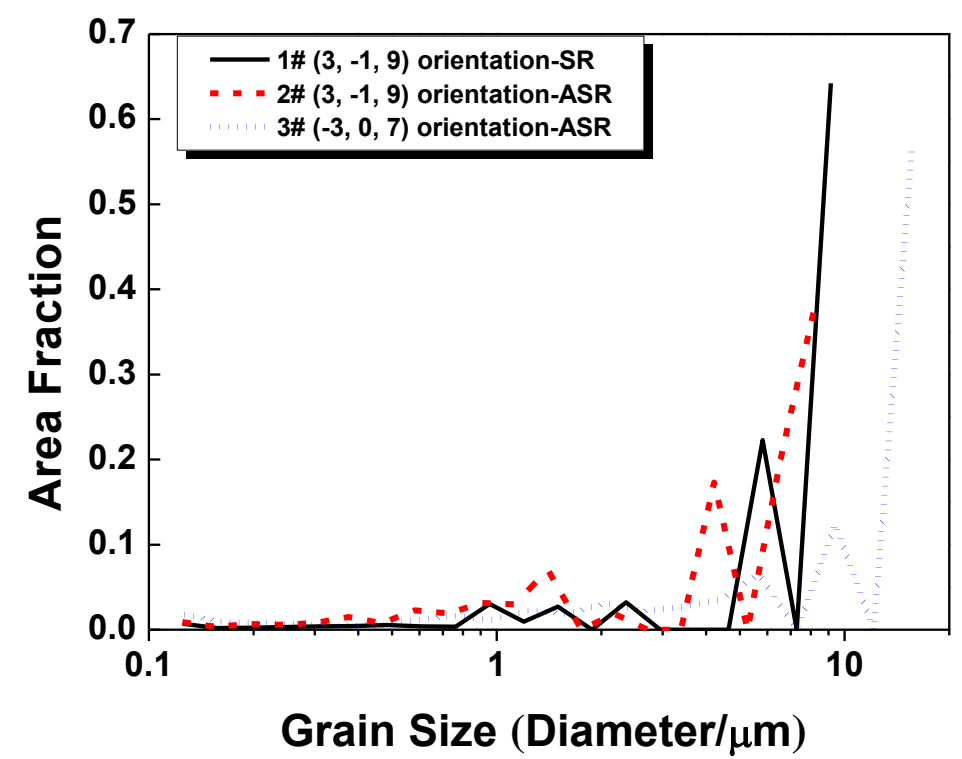

Fig. 4. Grain sizes of three rolled specimens, the peak value of horizontal ordinate represents the grain size in deformation bands.

\section{Discussion}

\subsection{Texture evolution of shear bands}

Micro-grains in rolled single crystal specimens have different size and rotation degrees for ASR and SR process, indicated from IPF maps Fig. 3a and 3b. In order to analyze the texture evolution which is associated with grain shapes and orientation rotated, (111) pole figures for three rolled specimens and their corresponding deformation areas were given in Fig. 5.

Rolling texture rotate from the whole single crystal of raw material to micro-grains appeared in deformation area. Microtextures evolution in different rolling conditions could be discussed by comparing the pole figures of whole detected area with deformation area. In the SR processed specimen (1\#, Fig. 5a), the texture of the deformation area is nearly the same with the whole detected area. In the ASR processed specimens (2\#, Fig. 5b and 3\#, Fig. 5c), texture evolution depends on initial orientations. For $2 \#$ with $(3,-1,9)$ plane orientation, texture rotate from initial orientation to near Copper component $(\{112\}<111>)$. But for 3\# single crystal with orientation near Copper component, texture did not rotated during ASR process. 
Orientations of raw single crystals were also marked in Fig. 5 to show the texture rotations clearly. Tough textures rotated different degrees for ASR processed 2\# and 3\# specimen, the final texture of deformation areas were both towards Copper component, which have not appeared in the SR processed 1\# specimen.
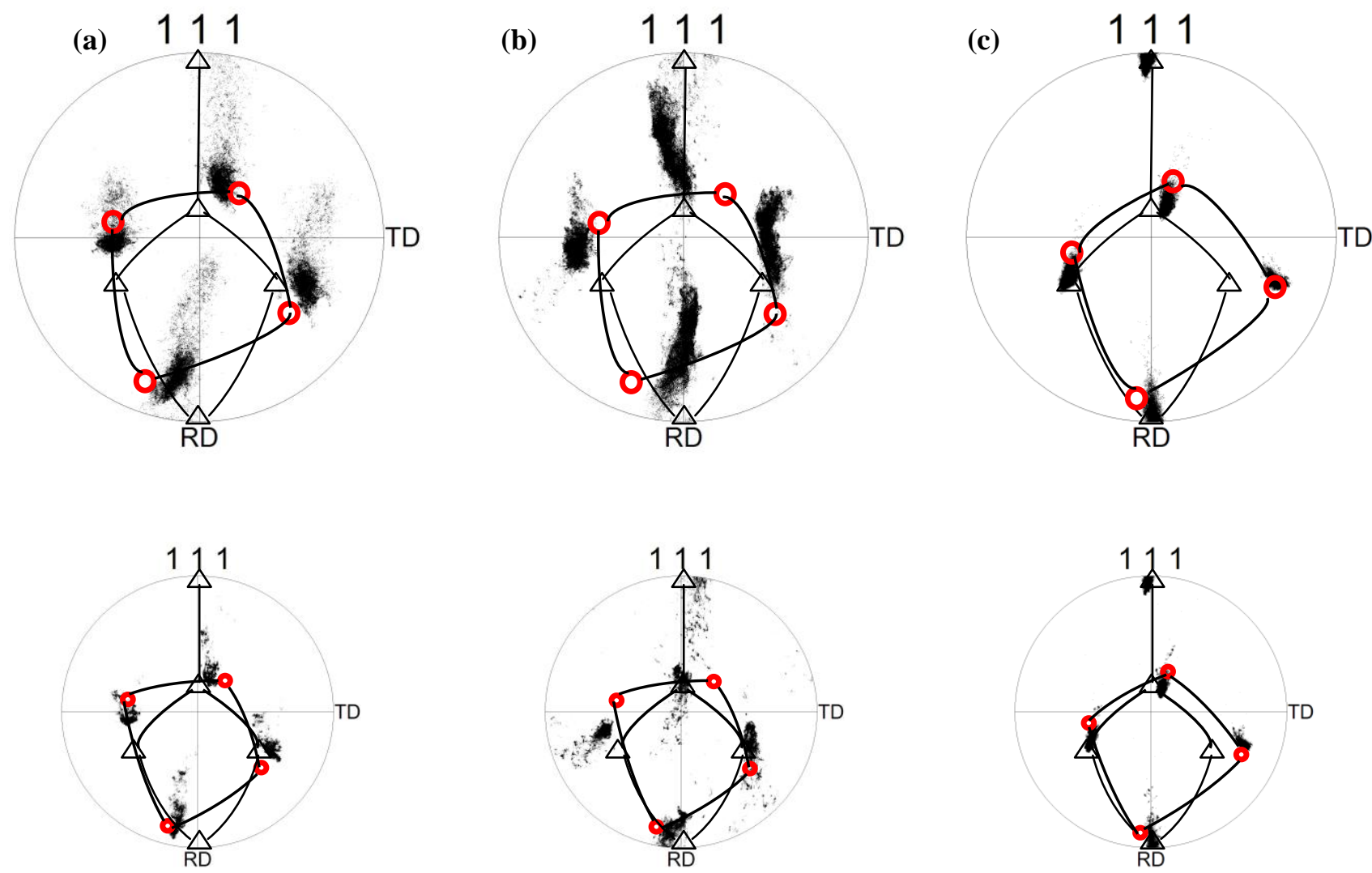

Fig. 5. (111) pole figures for three rolled specimens and their corresponding deformation areas: (a) 1\# specimen processed in SR; (b) 2\# specimen processed in ASR; (c) 3\# specimen processed in ASR. Orientations of raw single crystals were marked as red circle and Copper component was marked as black triangle in the pole figures.

\subsection{Texture components of microstructures}

As Copper component is significant in the textures of ASR processed specimens, marking the Copper component in EBSD maps is an effective way to study the texture components of microstructure in deformation bands. Copper component is marked as blue color in Fig. 6, and the component fraction is calculated by the OIM software. Results were listed in Table 1.

There is no Copper component appeared in SR processed specimens (Fig. 6a), 
single crystal was crushed into micro-grains and the grain hadn't rotated. For single crystal with the same orientation processed in ASR (Fig. 6b), texture component of the shear bands is almost filled with blue, which means the S-type grains in shear bands are mainly with Copper orientation. Compared with SR process, ASR process introduced additional shear strain during rolling [1], which induced shear bands appears more easily. Our results indicated that this shear strain in ASR induced grain to rotate towards Copper orientation, and this conclusion is well clarified by ASR processed 3\# specimen (Fig. 6c). 3\# specimen is the single crystal with orientation near Copper component, as the large blue color area in Fig. 6c showed. After processed in ASR, texture of shear bands remained Copper component. 


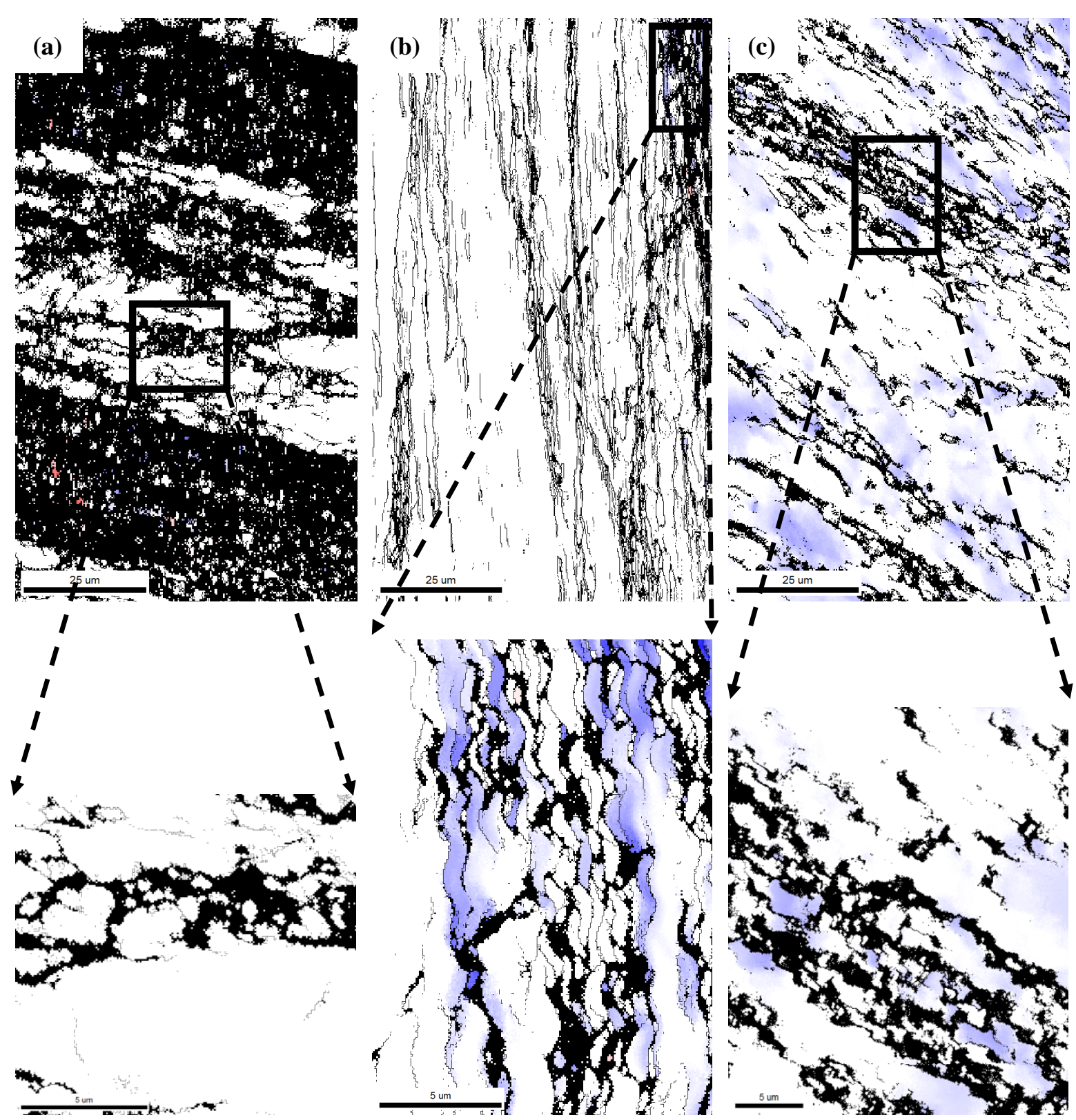

Fig. 6. Copper component $\{112\}<111>$ was marked as blue in the ND-Grain boundary maps for three rolled specimens: (a) $1 \#$ specimen processed in SR; (b) $2 \#$ specimen processed in ASR; (c) 3\# specimen processed in ASR.

Table 1 Copper component fraction in the ND-Grain boundary maps showed in Fig. 6 is calculated by the OIM software

\begin{tabular}{cccc}
\hline \multirow{2}{*}{ Conditions } & $\begin{array}{c}\text { SR for }(3,-1,9) \\
\text { orientation }(1 \#)\end{array}$ & $\begin{array}{c}\text { ASR for }(3,-1,9) \\
\text { orientation }(2 \#)\end{array}$ & $\begin{array}{c}\text { ASR for }(-3,0,7) \\
\text { orientation (3\#) }\end{array}$ \\
\hline \begin{tabular}{c} 
Copper component $\begin{array}{c}\text { fraction } \\
\text { frany }\end{array}$ \\
\cline { 2 - 4 }
\end{tabular} & $0.02 \%$ & $0.5 \%$ & $32.1 \%$ \\
\hline
\end{tabular}




\section{Conclusions}

(1) Single crystal specimens with the same crystallographic orientation were rolled in SR and ASR. ASR induced more homogeneous deformation compared with the SR process. Shear bands appeared in deformation area of ASR, while no grain rotations appeared in SR process.

(2) Single crystal specimens with different orientations were rolled in ASR. Microstructure of shear bands depends on the initial orientations of single crystals. After single crystal with $(3,-1,9)$ plane orientation is processed in ASR, shear bands consist of S-type grains were formed, which have uniform size and almost the same twisting degree. But for single crystal with $(-3,0,7)$ plane orientation, shear bands have various twisting degrees and grains distributed inhomogeneously.

(3) In the SR processed specimen, the texture of the deformation area is barely changed. In the ASR processed specimens, texture of the shear bands always rotated towards Copper component for single crystals with different plane orientation.

\section{Acknowledgements}

The authors gratefully acknowledge the support from the Chinese National Science Foundation (Project No. 51375503), the 973 program foundation of China (Grant No. 2012CB619505), the Major State Research Program of China (Project No. 2016YFB0300901) and the Major Science and Technology Project of Guangxi Province in China (Project No. 1412001-5).

\section{References}

[1] S. Wronski, B. Bacroix, Microstructure evolution and grain refinement in asymmetrically rolled aluminium, Acta Mater. 76 (2014) 404-412.

[2] S. Wronskia, B. Bacroixb, Texture and microstructure variation in asymmetrically rolled steel, Mater. Charact. 118 (2016) 235-243.

[3] Y. M. Hwang, G. Y. Tzou, Analytical and experimental study on asymmetrical sheet rolling, Int. J. Mech. Sci. 39 (1997) 289-303.

[4] J. Sidor, A. Miroux, R. Petrov, L. Kestens, Microstructural and crystallographic aspects of 
conventional and asymmetric rolling processes, Acta Mater. 56 (2008) 2495-2507.

[5] S. Wronski, K. Wierzbanowski, B. Bacroix, T. Chauveau, M. Wrobel, E. Rauch. Texture heterogeneity of asymmetrically cold rolled low carbo steel, Arch. Metall. Mater. 54 (2009) 89-102.

[6] M. R. Van Der Winden, Method for Processing a Continuously Cast Metal Slab or Strip, and Plate or Strip Produced in This Way; 2005. Patent No: US 20050000678.

[7] M. Shokoufeh, D. H. Peter, E. S. Nicole, B. H. Timothy, The role of shear banding on the fatigue ductility of ultrafine-grained aluminium, Scripta Mater. 68 (2013) 269-272.

[8] Y. ZUO, X. FU, J. CUI, X. TANG, L. MAO, L. LI, Q. ZHU, Shear deformation and plate shape control of hot-rolled aluminium alloy thick plate prepared by asymmetric rolling process, Trans. Nonferrous Met. Soc. China. 24 (2014) 2220-2225.

[9] S. Jurij, M. Alexis, P. Roumen, K. Leo, Microstructural and crystallographic aspects of conventional and asymmetric rolling processes, Acta Mater. 56 (2008) 2495-2507.

[10] B. Benoît, B. Somjeet, I. K. Dong, S. T. László, S. Satyam, Analysis of microstructure and texture evolution in pure magnesium during symmetric and asymmetric rolling, Acta Mater. 57 (2009) 5061-5077.

[11] O. Engler, J. Hirsch, Texture control by thermomechanical processing of AA6xxx Al-Mg-Si sheet alloys for automotive applications—a review, Mater. Sci. Eng. A. 336 (2002) 249-262.

[12] J. H. Han, J. Y. Suh, K. K. Jee, J. C. Lee, Evaluation of formability and planar anisotropy based on textures in aluminum alloys processed by a shear deforming process, Mater. Sci. Eng. A. 477 (2008) 107-120.

[13] A. Akef, J. H. Driver, Orientation splitting of cube-oriented face-centered cubic crystals in plane strain compression, Mater. Sci. Eng. A. 132 (1991) 245-255.

[14] K. Shiomi, K. Eiji, K. Masahiro, Deformation and recrystallization textures of copper single crystals and bicrystals, J. Mater. Sci. 27 (1992) 4927-4934.

[15] T. Kamijo, H. Adachihara, H. Fukutomi, Formation of a (001) [100] deformation structure in aluminum single crystals of an S-orientation, Acta Mater. 41 (1993) 975-985.

[16] K. Kashihara, H. Inagaki, Rolling textures in aluminum single crystal deviated by 5 degrees about rolling direction from (001) [100] orientation, Mater. Trans. 48 (2007) 1986-1991.

[17] User Manual of the HKL Channel 5 Software, Oxford Instruments, UK, 2010. 

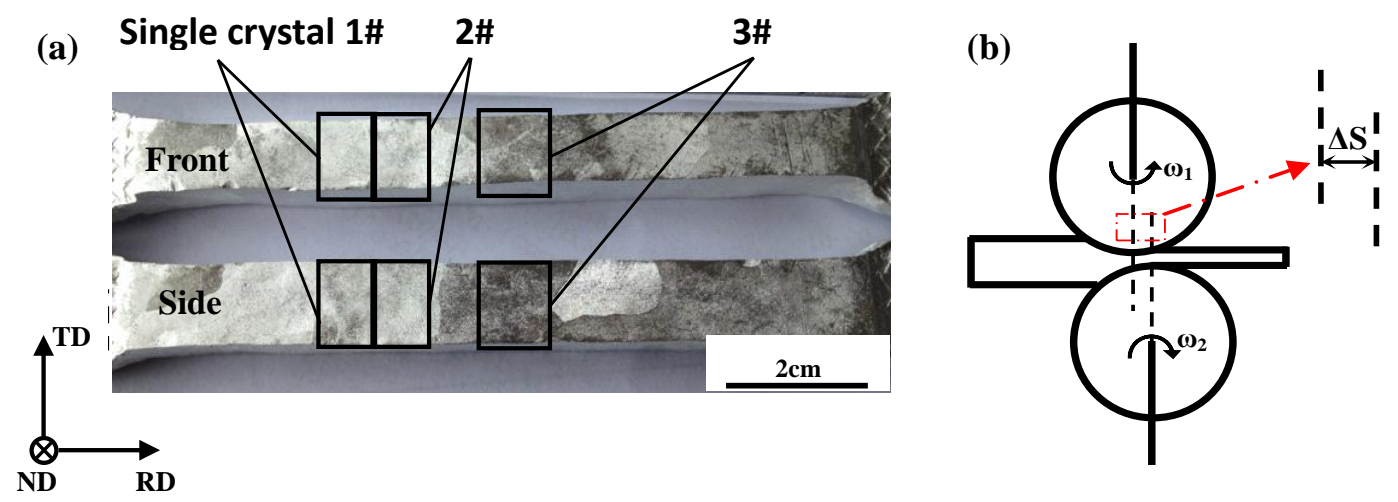

Fig. 1. (a) Large grains bar with single crystal specimens 1\#, 2\# and 3\#; (b) Schematic illustration of the rolling system designed for ASR process. 


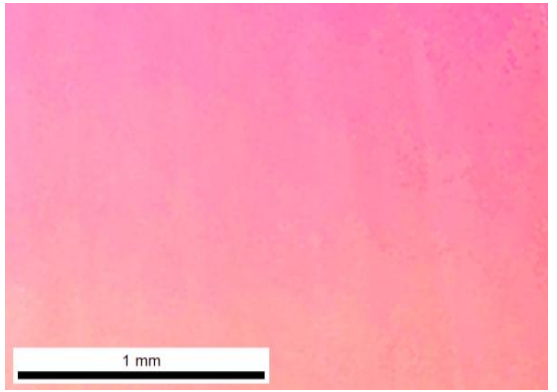

(a)

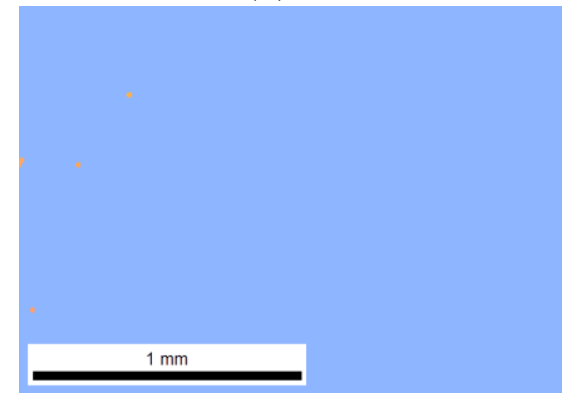

(c)

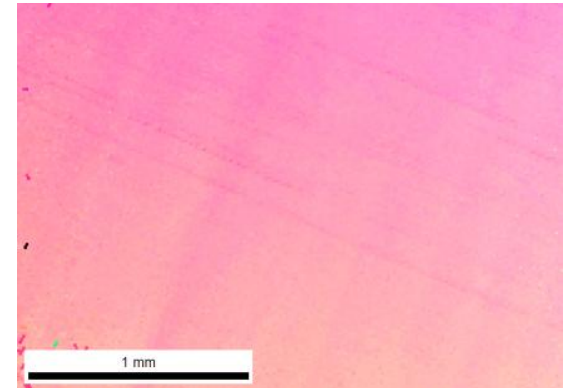

(b)

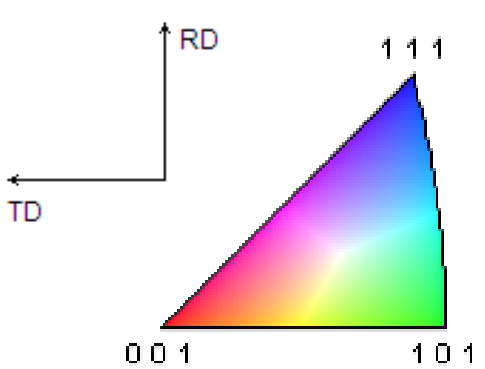

(d)

Fig. 2. The orientations of the Al-2Cu alloys single crystal specimens were determined by EBSD, (a) of $1 \#$ specimen and (b) of $2 \#$ specimen with (hkl) [uvw] orientation of $(3,-1,9)[-8,3,3]$; (c) of $3 \#$ specimen with (hkl) [uvw] orientation of $(-3,0,7)$ [7, -5, 3]; (d) Color coded map type of the [001] IPF in aluminum. 

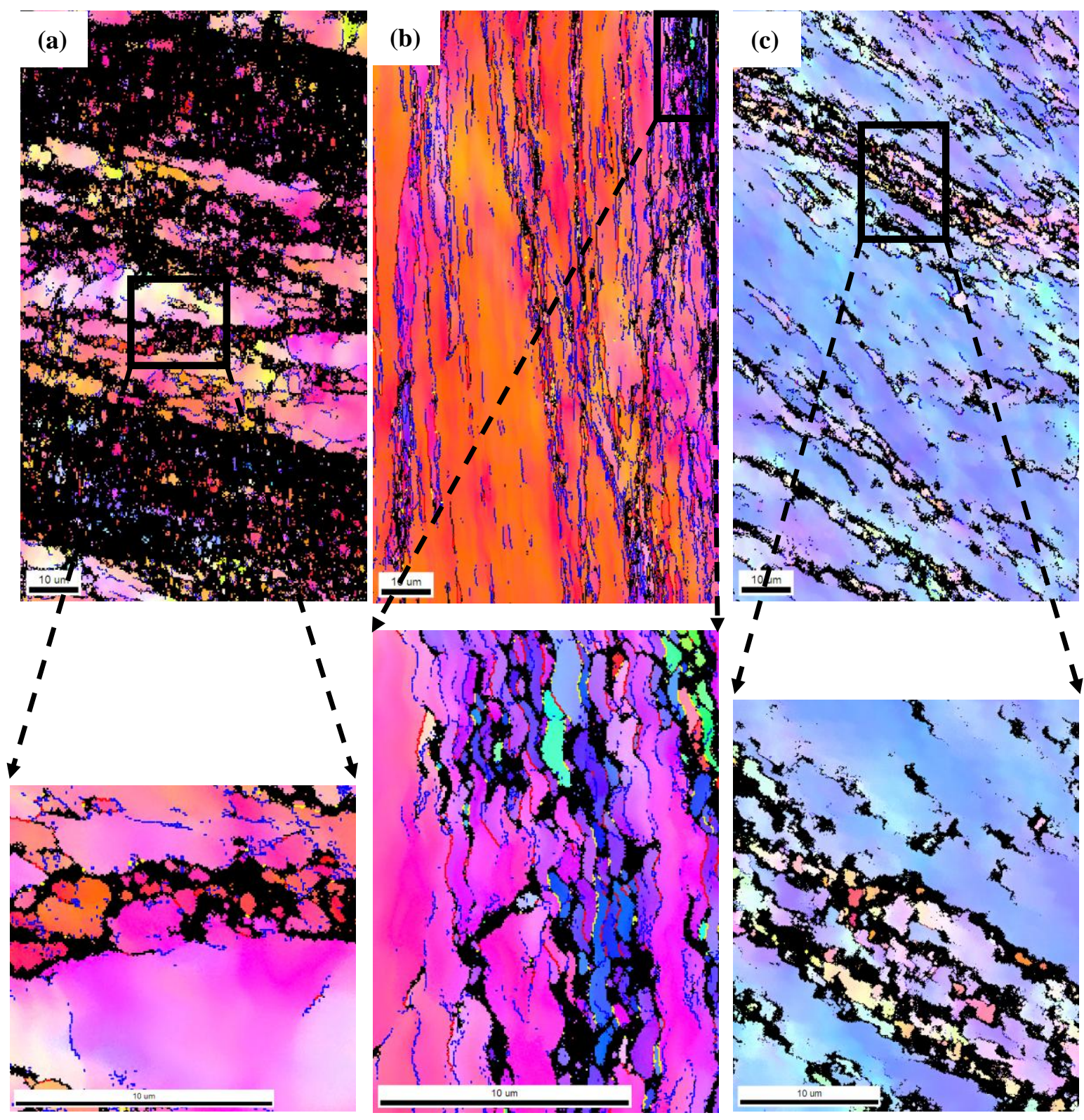

Fig. 3. ND-IPF maps for the three $\mathrm{Al}-2 \mathrm{Cu}$ alloys single crystal specimens processed in different rolling conditions: (a) 1\# specimen processed in SR; (b) 2\# specimen processed in ASR; (c) 3\# specimen processed in ASR. Rectangular areas were shown more clearly under the IPF maps. The grain boundaries were defined as low angle grains with $2^{\circ}-5^{\circ}$, sub-grains with $5^{\circ}-15^{\circ}$, and high angle grains with $15^{\circ}-180^{\circ}$, showed in blue, red and yellow colors respectively. 


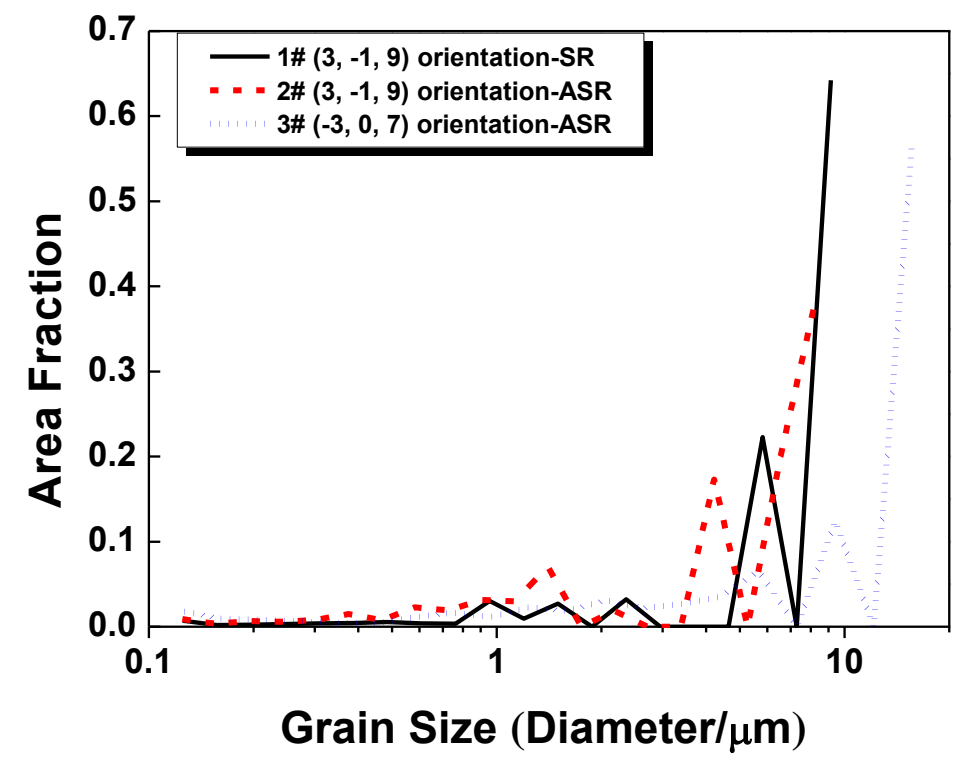

Fig. 4. Grain sizes of three rolled specimens, the peak value of horizontal ordinate represents the grain size in deformation bands. 

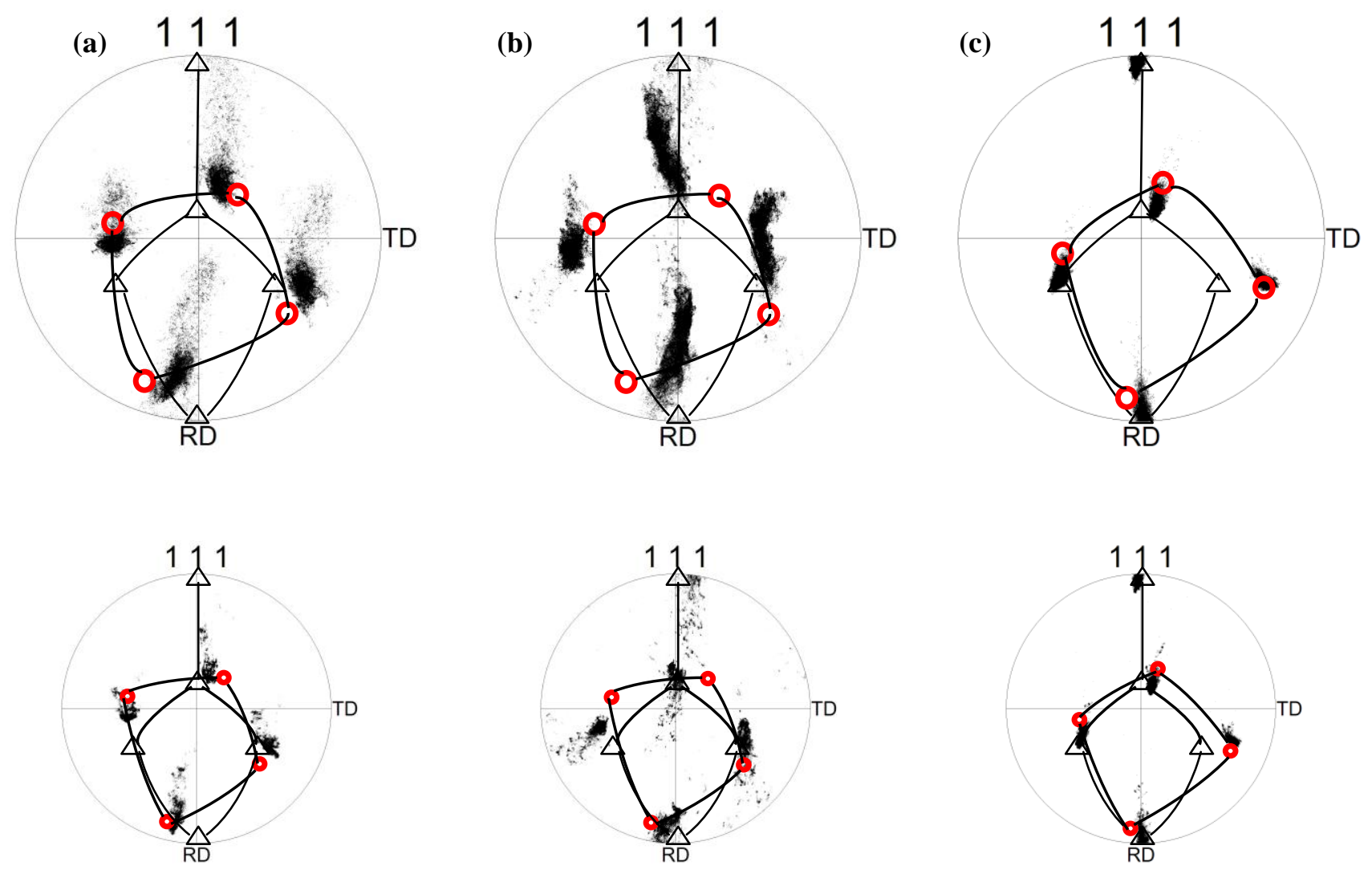

Fig. 5. (111) pole figures for three rolled specimens and their corresponding deformation areas: (a) 1\# specimen processed in SR; (b) 2\# specimen processed in ASR; (c) 3\# specimen processed in ASR. Orientations of raw single crystals were marked as red circle and Copper component was marked as black triangle in the pole figures. 


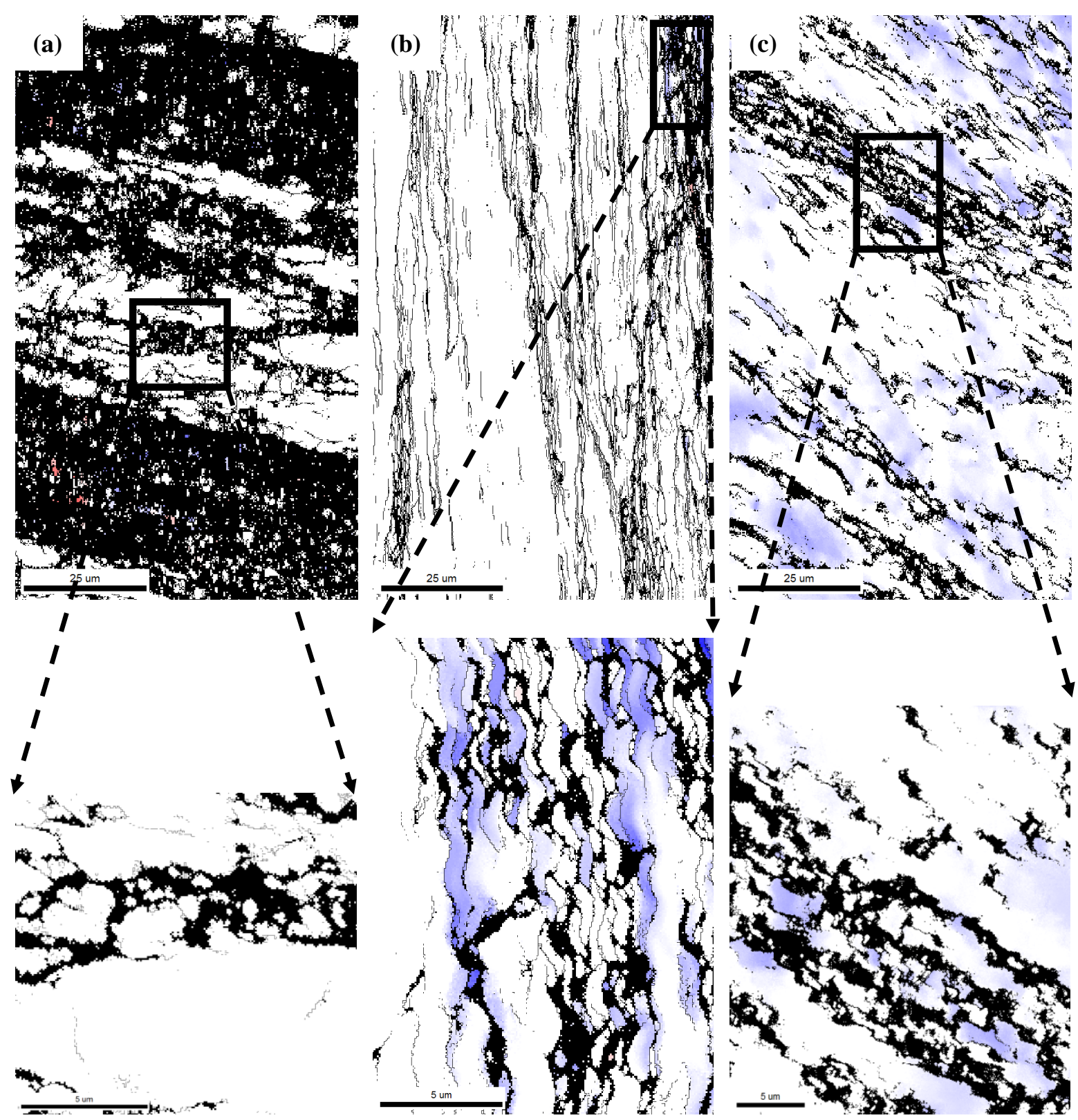

Fig. 6. Copper component $\{112\}<111>$ was marked as blue in the ND-Grain boundary maps for three rolled specimens: (a) 1\# specimen processed in SR; (b) 2\# specimen processed in ASR; (c) 3\# specimen processed in ASR. 
Table 1 Copper component fraction in the ND-Grain boundary maps showed in Fig. 6 is calculated by the OIM software

\begin{tabular}{cccc}
\hline & $\begin{array}{c}\text { SR for }(3,-1,9) \\
\text { orientation (1\#) }\end{array}$ & $\begin{array}{c}\text { ASR for }(3,-1,9) \\
\text { orientation }(2 \#)\end{array}$ & $\begin{array}{c}\text { ASR for }(-3,0,7) \\
\text { orientation (3\#) }\end{array}$ \\
\hline \multirow{2}{*}{$\begin{array}{c}\text { Copper component } \\
\text { fraction }\end{array}$} & $0.02 \%$ & $0.5 \%$ & $32.1 \%$ \\
\cline { 2 - 4 } & $0.01 \%$ & $25.0 \%$ & $18.8 \%$ \\
\hline
\end{tabular}



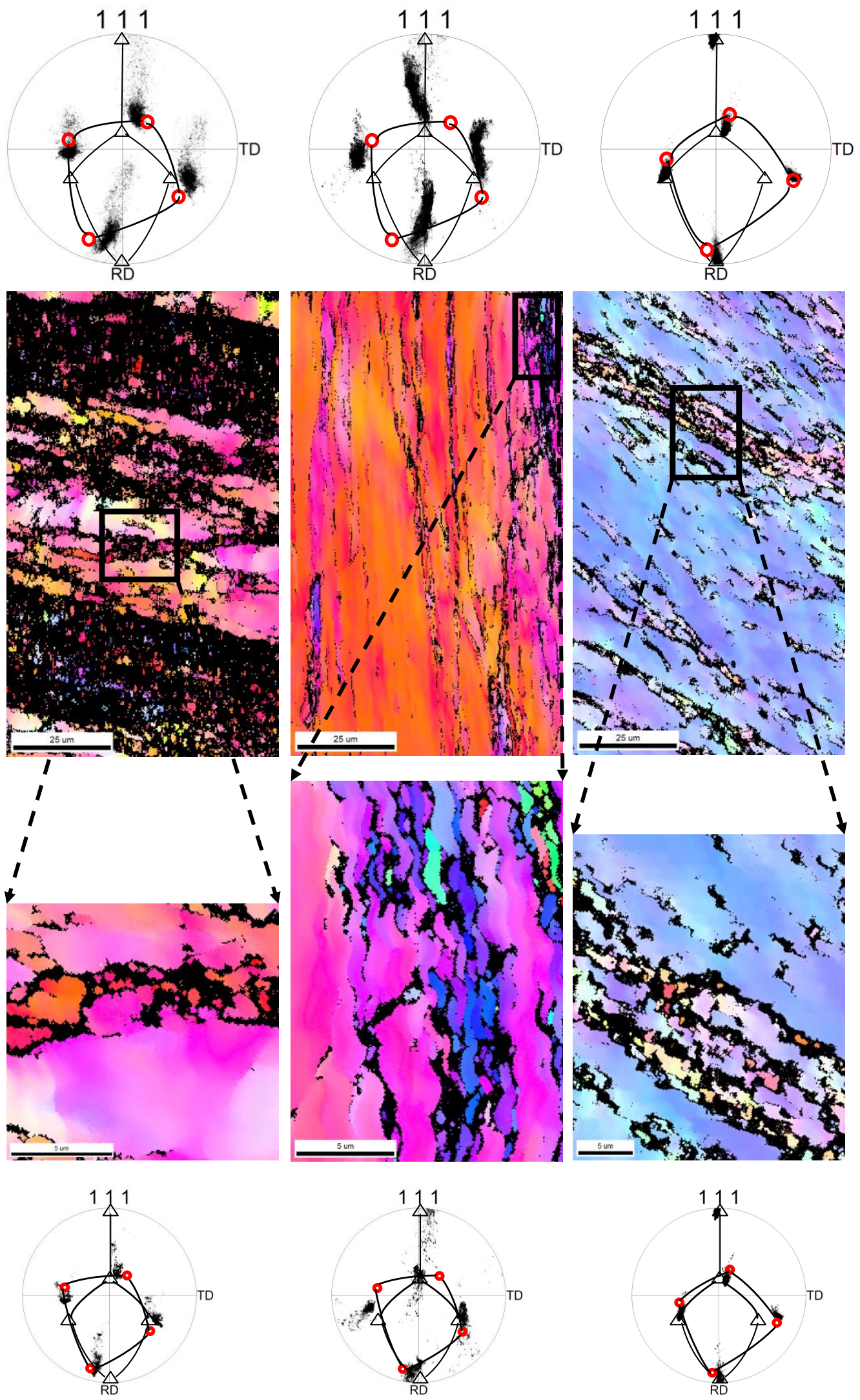